\title{
Estimating poisoning substance amounts: Comparative study of the accuracy of health care professionals and non-practitioners
}

\author{
LEE DONG HOON ${ }^{1}$, CHOI YOON HEE ${ }^{2}$, LEE DUK HEE ${ }^{2}$ \\ ${ }^{1}$ Department of Emergency Medicine, College of Medicine, Chung-Ang University \\ ${ }^{2}$ Department of Emergency Medicine, Ewha Womans University, Seoul, Korea. \\ Corresponding author: \\ Choi Yoon Hee \\ Ewha Womans University Medical Center 911-1 Mokdong Yangcheon-gu Seoul, Korea \\ E-mail:like-lemontea@hanmail.net emdhlee@cau.ac.krcalla521@empas.com
}

\section{ABSTRACT}

Objective. Intentional or unintentional substance intoxications are common in patients presenting to the Emergency Department (ED). When we treat intoxicated patients, it is important to know the amount of drug ingestion. We invested the actual amount of semi-quantitative term expressed by patients, and investigated the accuracy of amount estimates by the public and healthcare professionals.

Participants and interventions. 200 volunteers (86 health care providers and 114 non-practitioners) participated. Participants grabbed the 3 types of tablets $(5 \mathrm{~mm}$, $10 \mathrm{~mm}$, and $15 \mathrm{~mm}$ ) in handfuls and fistfuls and estimated the tablet amounts. Actual amounts were measured. 100 volunteers (58 health care providers and 41 non-practitioners) participated in the investigation of accuracy of liquid amount estimation. Participant ingested water in $2.6 \mathrm{~cm}$ diameter bottle in response to request to take 1 sip, 1 mouthful, and 3 sips.

Results. The estimated tablet counts became more accurate in terms of both fistfuls and handfuls as the size increased within the same shape classification. Participants tended to underestimate the counts of oval-shaped tablets to a greater extent than round tablets of the same size. The estimated liquids both groups of participants tended to underestimate the amounts but both groups overestimated the volumes when drinking 3 continuous sips. In tablets and liquids, there were no statistically significant differences in accuracy between the groups.

Conclusions. When approaching intoxicated patients who have visited emergency department (ED), treatment should be implemented based on the assumption that the actual ingested amounts are higher than the amounts estimated by patients.
Key-words: intoxication, tablets, amount, estimation, liquids

\section{KEY MESSAGES}

When the exact amount of pills was estimated, semi quantitative terms, such as 'handful' and 'mouthful' are used. Because those terms are subjective, we studied about how people estimate the amount of "handful" and evaluated their accuracy. As result, health care professionals and nonpractitioners both underestimated the amount of ingestion.

\section{INTRODUCTION}

Drug intoxication is among the reasons why patients visit the emergency department (ED). The causes of intoxication can be divided into intentional ingestion with suicidal purpose and accidental, nonintentional ingestion. In adults, most intoxication involves the excessive ingestion of toxic substances, or poisoning, whereas most pediatric cases are accidental. Intoxicated patients account for approximately $1 \%$ of all ED admissions, and the intoxication-related death toll is $0.5-1.4 \%$ of the total death toll, a fairly low rate. (1) However, Korea holds the record for the highest suicide rate among the Organization for Economic Co-operation and Development (OECD) countries in 2011, and the suicide rate exhibits a growing trend. According to the statistics, the mortality rate of drug intoxication with suicidal intention increased to $80 \%$. (2) Patients ingest various types of substances that mainly include drugs in tablet or liquid form, agricultural or industrial chemicals, or acidic or alkaline substances. In cases of accidental or intentional ingestion, accurate informa- tion about the amount of poisoning substance that was ingested is critical to the treatment of patients. However, it is very rare that patients who visit ED know the exact poisoning amount, and when patients are unable to answer questions because of unconsciousness, the amounts are usually determined from statements made by guardians or witnesses or from the estimations of physicians who provide treatment based on the remaining non-ingested amount. If the poisoning substance is liquid, the poisoning amount is estimated by the remaining amount in the container or, if a witness observed the patient's consumption of the substance, the quantity is estimated in units of mouthfuls or gulps. For substances in tablet form, the maximum number of ingested tablets can be estimated based on the prescribed amount or the remaining number of tablets. However, in cases involving drugs sold in large storage containers, such as Tylenol ${ }^{\circ}$ or Ad$\mathrm{vil}^{\star}$, or in cases involving hypnotics, sedative drugs, or anti-psychotic drugs that patients might save over time and ingest at once, the amounts of ingested substances are mostly described in terms of handfuls. Nonetheless, no studies have evaluated the accurate quantities of mouthfuls and handfuls frequently used to estimate the poisoning amounts ingested by patients. In addition, there have been no investigations or data regarding the amounts considered by patients and health care professionals to comprise mouthfuls or handfuls and the accuracy of these amounts.

In the present study, we firstly determined the actual amounts of the semi-quantitative term used by patients and secondly investigated the accuracies of the amounts estimated by members of the public and healthcare professionals. 


\section{SUBJECTS AND METHODS}

\section{Research participants}

The participants in the present study were healthy adults who had no swallowing or hand grip difficulties, understood the purpose of the study, and agreed to voluntarily participate. The volunteers were classified as either non-practitioners or health care professionals. This was approved by the institutional review board of Ewha university medical center (EUMC 2015-10-025-002).

\section{Measurement of tablet quantities}

Tablet preparation and measurement

Tablets were divided into 3 types according to length $(5 \mathrm{~mm}, 10 \mathrm{~mm}$, and 15 $\mathrm{mm})$. The study used $5-\mathrm{mm}$ round tablets (Aron ${ }^{\circledR}$, doxylamine succinate $25 \mathrm{mg}$, Korea Alicopharm Co., Ltd.), 10-mm round tablets (Aspirin ${ }^{\circledR}$, acetylsalicylic acid 500 mg, Bayer Korea Ltd., Kyunggi-do, Korea), 10-mm oval tablets (Xanax ${ }^{\oplus}$, alprazolam $0.25 \mathrm{mg}$, Pfizer Pharmaceuticals Korea, Seoul, Korea), and 15-mm elliptical tablets $\left(\right.$ Tylenol $^{\oplus}$, acetaminophen $500 \mathrm{mg}$, Yansen Korea Ltd.) were used. Participants grabbed the tablets in handfuls, defined as the maximum amount that could be held in 1 hand, and fistfuls, defined as the maximum amount that could be held completely within 1 hand (i.e., not visible outside of the hand), and then placed the tablets on the floor. Finally, they wrote down their estimations of the numbers of tablets that they had grabbed (figure 1). After the participants completed the experiments, the examiner counted the actual numbers of tablets; participants were not informed about the actual amounts of drugs measured during the experiment.

\section{Measurement of liquid amounts}

Types of container and liquids

As the internal dimeter of a container opening can affect the amount of a sip or mouthful, experiments were performed using a medical bottle with a $2.6-\mathrm{cm}$ internal diameter. Graduations on the medical bottles were hidden with opaque paper to avoid biasing the participants' estimations, and the experiments were performed with bottles containing $300 \mathrm{~mL}$ of room-temperature $\left(22-23^{\circ} \mathrm{C}\right)$ water.

Liquid amount measurement methods While seated comfortably, the participants drank water from 3 medicine bottles containing unknown amounts. The participants took a sip of water from the first bottle, a mouthful of water from the second bottle, and 3 continuous drinks of water without pausing from the third bottle. Participants recorded in writing the estimated amounts of liquid consumed, and the remaining water in each container was measured using a mass cylinder after the participants left the room.

\section{Data analysis}

Estimation of the sample size to analyze these data was performed using $\mathrm{G}^{\star}$ Power software version 3.1.3 (Franz Faul, Universität Kiel, Germany). (3) In light of a previous pilot study, the effect size calculated was 0.55 . (4) The minimum sample size required was 106 for a two-tailed Student's $\mathrm{t}$ test with a significance level of 0.05 and statistical power of 0.80 .

Chi-square was performed for non-continuous variables, and Student t-test was used for continuous variables using PASW version 18 (SPSS, Inc., Chicago, IL, USA). An analysis of variance (ANOVA) with Bonferroni correction was performed (as

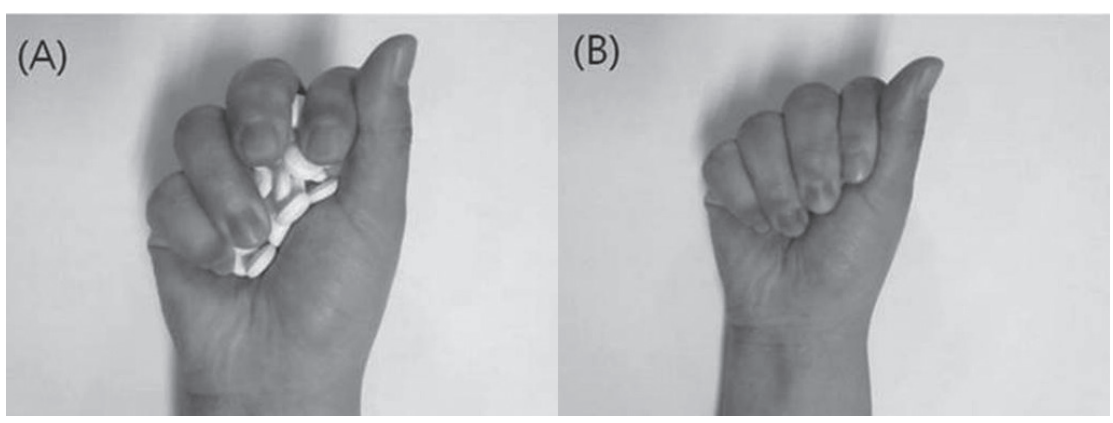

Figure 1. Manner of holding. (A) Handful and (B) fistful.

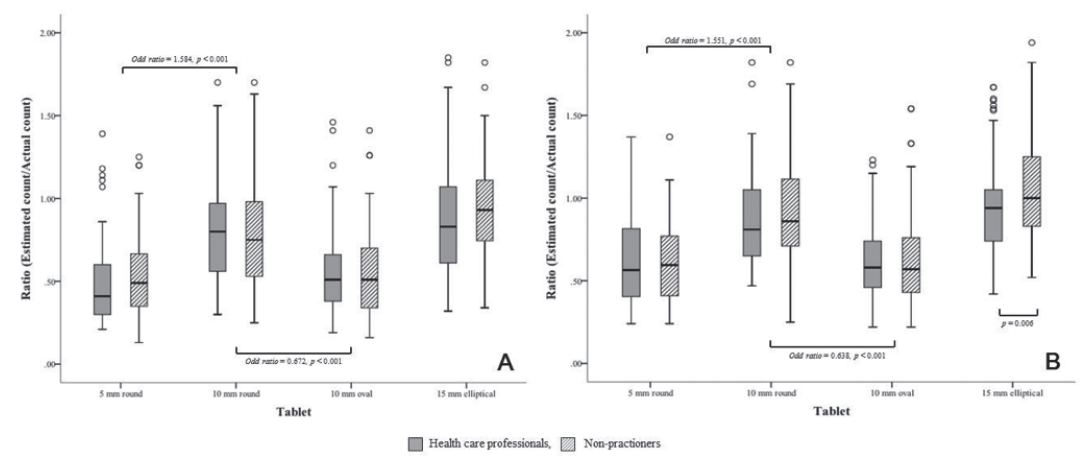

Figure 2. Comparison of estimation of tablet count according to size and shape. (A: Handful grip, B: Fistful grip)

The estimated tablet counts became more accurate as the size increased within the same shape classification ( $p<0.001,5 \mathrm{~mm}$ vs. $10 \mathrm{~mm}$ round-shaped tablet, A and B). Participants underestimated the counts of oval-shaped tablets than round-shape tablets of the same size ( $p<0.001,10 \mathrm{~mm}$ round-shaped vs. oval-shaped tablets, A and B). There was no statistically significant difference in accuracy between the groups except for 15- $\mathrm{mm}$ elliptical tablets when fistful grip $(p=0.006, B)$. 
shaped tablets, figure 2). There was no statistically significant difference in accuracy between the groups except for $15-\mathrm{mm}$ elliptical tablets when fistful grip ( $\mathrm{p}=0.006$, table 1, figure 2). When a fistful grip was used, non-medical personnel overestimated the amount of 15-mm elliptical tablets. With smaller pills, the self-estimated amount was less than the actual amount. Additionally, participants reported smaller amounts when grabbing fistfuls than when grabbing handfuls (table 1).

\section{Liquid medicine}

One hundred participants were involved. The mean participant age was $27.33 \pm 3.16$ years. The participants were 58 health care providers (16 men, 42 women) and 41 non-practitioners (12 men, 29 women). There were no statistically significant differences between the health care providers and non-practitioners $(\mathrm{p}=0.855)$.

There were no statistically significant differences in accuracy between the groups (table 2). The 1 sip amounts were 30.00 $\pm 15.28 \mathrm{~mL}$ for health care providers and $26.46 \pm 12.76 \mathrm{~mL}$ for non-practitioners ( $\mathrm{p}$ $=0.228$ ), and the mouthful amounts of liquid were $60.09 \pm 17.28 \mathrm{~mL}$ for health care providers and $59.63 \pm 20.17 \mathrm{~mL}$ for nonpractitioners $(\mathrm{p}=0.952)$. However, both groups of participants tended to underestimate the amounts when drinking a sip of liquid and overestimate the volumes when drinking 3 continuous sips (table 2).

Table 1. Comparison of estimation for tablet count between health care professionals and non-practitioners

\begin{tabular}{|c|c|c|c|c|c|c|c|}
\hline & \multicolumn{3}{|c|}{ Health care professionals } & \multicolumn{3}{|c|}{ Non-practitioners } & \multirow[t]{2}{*}{ p-value for ratio } \\
\hline & Estimated coun & Actual count & ratio & Estimated coun & t Actual count & ratio & \\
\hline \multicolumn{8}{|c|}{$5 \mathrm{~mm}$ round tablet } \\
\hline Handful grip & $137.12 \pm 93.29$ & $293.33 \pm 131.00$ & $0.48 \pm 0.24$ & $104.62 \pm 76.95$ & $221.45 \pm 136.48$ & $0.53 \pm 0.23$ & 0.182 \\
\hline Fistful grip & $75.47 \pm 57.56$ & $122.45 \pm 54.61$ & $0.65 \pm 0.34$ & $60.42 \pm 48.90$ & $99.30 \pm 51.73$ & $0.64 \pm 0.29$ & 0.844 \\
\hline \multicolumn{8}{|c|}{$10 \mathrm{~mm}$ round tablet } \\
\hline Handful grip & $51.81 \pm 28.48$ & $70.41 \pm 36.80$ & $0.80 \pm 0.29$ & $60.84 \pm 39.88$ & $83.67 \pm 44.77$ & $0.80 \pm 0.34$ & 0.995 \\
\hline Fistful grip & $27.94 \pm 14.22$ & $34.12 \pm 16.56$ & $0.87 \pm 0.28$ & $31.10 \pm 16.09$ & $35.48 \pm 15.50$ & $0.91 \pm 0.30$ & 0.274 \\
\hline \multicolumn{8}{|c|}{$10 \mathrm{~mm}$ oval tablet } \\
\hline Handful grip & $91.12 \pm 53.12$ & $180.74 \pm 99.56$ & $0.57 \pm 0.27$ & $109.17 \pm 82.53$ & $216.55 \pm 118.32$ & $0.55 \pm 0.26$ & 0.693 \\
\hline Fistful grip & $48.62 \pm 28.56$ & $84.55 \pm 43.69$ & $0.61 \pm 0.23$ & $55.76 \pm 36.03$ & $93.45 \pm 45.57$ & $0.64 \pm 0.35$ & 0.461 \\
\hline \multicolumn{8}{|c|}{15 mm elliptical tablet } \\
\hline Handful grip & $39.97 \pm 19.27$ & $51.45 \pm 26.67$ & $0.86 \pm 0.32$ & $45.36 \pm 31.10$ & $53.41 \pm 33.28$ & $0.92 \pm 0.28$ & 0.162 \\
\hline Fistful grip & $21.87 \pm 11.03$ & $24.08 \pm 9.98$ & $0.94 \pm 0.31$ & $23.64 \pm 13.27$ & $22.20 \pm 9.30$ & $1.07 \pm 0.32$ & 0.006 \\
\hline
\end{tabular}

There was no statistical significant difference between health-care professionals and non-practitioners except $15 \mathrm{~mm}$ elliptical tablet. $\left({ }^{*}: \mathrm{p}<0.05\right)$

Table 2. Comparison of estimation for liquid amount between health care provider and non-medical personnel.

\begin{tabular}{llll}
\hline & Health care professionals & Non-practitioners & p-value \\
\hline $\mathbf{1}$ sip & & & \\
\hline self-estimated amount & $19.38 \pm 12.67$ & $20.78 \pm 12.45$ & 0.586 \\
\hline actual amount & $30.00 \pm 15.28$ & $26.46 \pm 12.76$ & 0.228 \\
\hline ratio (estimated: actual) & $0.73 \pm 0.44$ & $0.86 \pm 0.48$ & 0.380 \\
\hline Mouthful & & $55.61 \pm 23.43$ & 0.907 \\
\hline self-estimated amount & $60.26 \pm 27.36$ & $59.63 \pm 20.17$ & 0.952 \\
\hline actual amount & $60.09 \pm 17.88$ & $0.98 \pm 0.40$ & 0.671 \\
\hline ratio (estimated: actual) & $1.03 \pm 0.40$ & & 0.177 \\
\hline $\mathbf{3}$ sips & & $74.88 \pm 33.02$ & 0.536 \\
\hline self-estimated amount & $74.48 \pm 31.51$ & $64.27 \pm 26.52$ & 0.675 \\
\hline actual amount & $66.55 \pm 26.11$ & $1.22 \pm 0.46$ & \\
\hline ratio (estimated: actual) & $1.18 \pm 0.46$ & & \\
\hline
\end{tabular}

\section{DISCUSSION}

Drug intoxication is among the causes of emergency room visits. According to the ED-based injury surveillance in 2010,
1,787 deaths were related to intoxication, of which $31.3 \%$ were accidental intoxication, $59.3 \%$ were suicidal, and $9.4 \%$ were of unidentified intention. (2) In 2014, people who died of intoxication were 1,559 in the USA, and intentional intoxication accounted for 1064. (5) Among reported fatalities, the first ranked substance was a pharmaceutical such as analgesics, simulants/street drugs, cardiovascular drugs, 
antidepressants, and sedative/hypnotic/ antipsychotics. In the USA, the damage caused by intoxication is mostly asymptomatic or non-fatal, whereas in Korea the proportion of seriously intoxicated patients is very high. According to a 2009 ED-based intoxication survey, the admission and mortality rates of intoxicated patients in Korea were $29.55 \%$ and $4.4 \%$, respectively. (2) Knowledge about the amounts of poisoning or doses ingested by intoxicated patients is very important because these amounts are used for drug toxicity severity evaluations or as a predictive factor for death. As convenience stores in Korea have been allowed to sell medicines for which prescriptions are not required since November 2011, it has become possible for non-practitioners to purchase large quantities of medicines. Medicines that can be purchased without prescriptions are mostly antipyretic analgesics such as Tylenol $^{\circ}$ tablets, Brufen ${ }^{\oplus}$ Syrup, and digestive medicines, but these medicines also include some sleep inducers (doxylamine). Medicines that do not require prescriptions from physicians or medication counselling from pharmacists tended to be considered as non-harmful to the body, and people from a wider range of ages can easily purchase these medicines in large quantities. In the context of drug intoxication, these medicines could become more harmful. Amounts of medicines that are sold in large storage containers or collected without timely ingestion by patients and subsequently ingested at once are generally described in handfuls or fistfuls. However, no previous studies have evaluated the actual amounts of tablets corresponding to ingested amounts measured in handfuls or fistfuls. In the present study, the semi-quantitative amounts of a handful and fistful were actually measured. In addition, the amounts of the semi-quantitative terms that had been estimated by health care professionals and non-practitioners estimated were accurately measured to measure the differences between the estimated and actual amounts. In this study, tablets usually ingested by subjects were classified into 3 types according to the length: $5 \mathrm{~mm}, 10 \mathrm{~mm}$, and $15 \mathrm{~mm}$ (table 1). The average numbers of 5-mm-size tablets per fistful were $122.45 \pm 54.61$ for health care professionals and $99.30 \pm 51.73$ for non-practitioners; average numbers of the same-sized tables per handful were $293.33 \pm 131.00$ for health care professionals and $221.45 \pm 136.48$ for non-practitioners. The average numbers of $10-\mathrm{mm}$ round tablets per fistful were $34.12 \pm 16.56$ for health care professionals and $35.48 \pm 15.50$ for non-practitioners; the average numbers per handful for the same groups were 70.41 \pm 36.80 and $83.67 \pm 44.77$, respectively. The average numbers of 10 -mm oval tablets per fistful were $84.55 \pm 43.69$ for health care professionals and $93.45 \pm 45.57$ for nonpractitioners, whereas the average numbers per handful were $180.74 \pm 99.56$ and $216.55 \pm 118.32$, respectively. The average numbers of $15-\mathrm{mm}$ tablets per fistful were $24.08 \pm 9.98$ for health care professionals and $22.20 \pm 9.30$ for non-practitioners; the average numbers per handful were 51.45 \pm 26.67 and $53.41 \pm 33.28$, respectively. The estimated tablet counts in both fistfuls and handfuls became more accurate as the tablet size increased within the same shape type (figure 2). Participants tended to underestimate the counts of oval-shaped tablets to a greater extent than round tablets of the same size (figure 2). There were no statistically significant differences in accuracy between the groups except for 15-mm elliptical tablets (table 1). It is possible that the actual amount of ingested small-sized tablets may be greater than that described by the patient or caregiver upon visiting the emergency department. In previous studies, estimated volumes and dimensions were investigated in other settings, including relative to blood loss, body size, and tissue volume, and were found to be prone to error, particularly underestimation (6-9) In this study, health care professionals tended to underestimate the counts of $15-\mathrm{mm}$ tablets. According to previous studies, the propensity of doctors to underestimate blood loss was reported across a range of medical and surgical specialties. $(7,10)$ However, we do not suggest that physicians should simply ignore the amounts of ingested tablets estimated by patients and caregivers.

It was found that when collecting a fistful, participants would grab twice as many tablets than were held in a handful, indicating that even when the same-sized hands are used for measurement, the number of tablets can differ greatly depending on the holding method. Detailed information, including the method used by patients when holding tablets, should be investigated to more accurately determine the amount of ingested drugs.

Estimations of poisonous and toxic liquid substances also depend on statements provided by patients or guardians. Quantitative estimations of the poisoning amounts made by the patients themselves and estimations based on the remaining amount in the container tend to underestimate the actual poisoning amounts and remaining amounts. (11) In general, poisoning amounts are determined by multiplying the estimated amount of a sip by the number of swallows. Previous estimates of sip amounts were generally calculated by having healthy American adults drink $175 \mathrm{~mL}$ of cold water from a cup and then dividing $175 \mathrm{~mL}$ by the number of swallows; the calculated mean sip amounts were 21 $\mathrm{mL}$ for male and $14 \mathrm{~mL}$ for female participants. (12) At present, the poisoning amounts in a Korean study of agricultural chemical intoxication were calculated by multiplying the patient-reported number of swallows by the estimated sip amount $(20-30 \mathrm{~mL}) .(13,14)$ To create a situation similar to actual agricultural chemical poisoning, a medicine bottle with a $2.6-\mathrm{cm}$ internal diameter, which is similar to the sizes of agricultural chemical bottle openings, was used in the present study. Previous studies were performed using liquids contained in cups; however, agricultural chemicals and other industrial liquids are contained in special containers with internal diameters smaller than that of a cup, and toxicologically, the tastes of these chemicals differed from that of water. As the container diameter becomes smaller, the flow amount decreases and negative pressure is formed in the container, thus decreasing the mean volume of 1 swallow. (15) When healthy American adults were tested using a chemical container with a $1.5-\mathrm{cm}$ diameter, the mean maximum sip volumes were $14.9 \mathrm{~mL}$ for men and 14.3 $\mathrm{mL}$ for women, which were smaller than the volumes measured when using a cup. (14) In contrast, although containers with a $2.6-\mathrm{cm}$ internal diameter were used in the present study, the poisoning agent sip amounts were $30.00 \pm 15.28 \mathrm{~mL}$ for health care providers and $26.46 \pm 12.76 \mathrm{~mL}$ for non-practitioners, which were higher than the results of the previous study (table 2). The poisoning agent mouthful amounts were $60.09 \pm 17.28 \mathrm{~mL}$ for health care providers and $59.63 \pm 20.17 \mathrm{~mL}$ for non-practitioners. Regarding liquid measurements, sip amounts tended to be underestimated, whereas the amount ingested during 3 continuous sips tended to be overestimated by both groups. In addition, it should be considered that drinking 1 sip or continuous sips would affect the swallowed amount. In a study on the effect of continuous sipping on the swallowed amount, as the number of sips increased from 1 sip to 5 sips and in particular from 1 sip to 3 sips, the amount per sip decreased. (16) When the amount of 1 sip was calculated by dividing the amount drank during 3 continuous sips by the number of sips, the amounts per sip decreased to $22.18 \pm 8.70 \mathrm{~mL}$ for health 
care providers and $21.42 \pm 8.84 \mathrm{~mL}$ for non-practitioners. The amount of a liquid mouthful was approximately 2 -fold higher than that of a sip, indicating that that amount of liquid poisoning ingested by patients could differ by as much as 2 -fold, depending on the drinking method. Therefore, for patients with liquid substance poisoning, it is necessary to obtain information about how many sips of the poisoning liquid were ingested and how the liquid was swallowed

\section{LIMITATIONS}

First, as the participants were limited to healthy adult men and women, some age groups were not included. The mean swallowed amounts might be lower for elderly patients. (17) Considering that the incidence of drug intoxication in the elderly has increased gradually and that half of all drug intoxications were caused by agricultural chemicals studies of swallowing amounts specific to elderly patients are needed.

Second, this study only considered the tab- let lengths with respect to size, but did not consider the tablet volumes and shapes. The number of tablets that could be held in a subject's hand would likely differ if tablets with the same lengths but different volumes and shapes were used. In this study, the results differed even between round and oval-shaped 10-mm tablets. Therefore, additional studies that consider the tablet volume, shape, or thickness are needed.

Third, the education level of the subjects is expected affect the estimation amounts; however, all of the participants in this study had graduated from university. Therefore, studies should be conducted in various population clusters.

Fourth, although differences in the tastes of liquids might influence the swallowed amount, only water, which is easy to consume, was used in the present study. In a study on taste-dependent swallowing action, the swallowing action was affected by bitter or salty tastes. For example, the swallow rates became slower and the interswallow interval (ISI), the value calculated by dividing the time taken to finishing consuming a liquid volume by the number of swallows, became longer. Taste was also found to affect swallowing capacity, or the consumed liquid volume divided by the number of swallows. (18) As most agrichemical or industrial substances have volatile or repulsive smells, liquids with tastes and smells that differ from those of the physiological saline solution used in the present study should be considered in additional studies on this issue.

\section{CONCLUSION}

When approaching intoxicated patients who have visited ED, treatment should be implemented based on the assumption that the actual ingested amounts are higher than the amounts estimated by patients, and preparation should be made for fatal situations. In addition, as the poisoning amounts might differ depending on the ingestion method, it is necessary to obtain a more in-depth medical history regarding the poisoning method when treating intoxicated patients.

\section{REFERENCES}

1. Korean Statistical Information Service. Cause of death. Deaths. Daejeon: Statistics Korea; 2015. Accessed 21 May, 2015 http://kosis.kr/statHtml/statHtml.do?orgId=101\&tblId=DT_1B34E02\&vw_cd=MT_ZTITLE\&list_id=D11\&seqNo=\&lang_ mode=ko\&language $=$ kor\&obj_var_id=\&itm_id=\&conn_path=E1\#

2. Injury surveillance report. Center for national injury surveillance system management and cooperation. Accessed 25 November, 2014 http://injury .cdc.go.kr/injury/

3. Faul F, Erdfelder E, Buchner A, Lang AG. Statistical power analyses using G*Power 3.1: test for correlation and regression analyses. Behav Res Methods 2009;41:1149-60.

4. Choi HS, Choi YH. Accuracy of tablet counts estimated by members of the public and healthcare professionals. Clin Exp Emerg Med 2015;2:168-73.

5. Mowry JB, Spyker DA, Brooks DE, McMillan N, Schauben JL. 2014 Annual Report of the American Association of Poison Control Centers' National Poison Data System (NPDS): 32nd Annual Report. Clin Toxicol 2015;53:962-1147.

6. Yoong W, Karavolos S, Damodaram M, Madgwick K, Milestone N, Al-Habib A, et al. Observer accuracy and reproducibility of visual estimation of blood loss in obstetrics: how accurate and consistent are health-care professionals? Arch Gynecol Obstet 2010;281:20713.

7. Toledo P, McCarthy RJ, Hewlett BJ, Fitzgerald PC, Wong CA. The accuracy of blood loss estimation after simulated vaginal delivery. Anesth Analg 2007;105:1736-40.

8. Greene S, Dargan P, Shin GY, Jones AI. Doctors and nurses estimation of the weight of patients: A preventable source of systematic error. J Toxicol Clin Toxicol 2004;42:611-5.

9. Schuld J, Kollmar O, Seidel R. Black C, Schilling MK, Richter S. Estimate or calculate? How surgeons rate volumes and surfaces. Langenbecks Arch Surg 2012;397:763-9.

10. Beer HL, Duvvi S, Webb CJ, Tandon S. Blood loss estimation in epistaxis scenarios. J Laryngol Otol 2005;119:16-8.

11. Hitchings AW, Wood DM, Warren-Gash C, Gil Rivas S, Dargan PI. Determining the volume of toxic liquid ingestions in adults: accuracy of estimates by healthcare professionals and members of the public. Clin Toxicol 2013;51:77-82.

12. Jones DV, Work CE. Volume of a swallow. Am J Dis Child 1961;102:427.

13. Cha YS, Kim H, Cho NH. Pyrethroid poisoning: features and predictors of atypical presentations. Emerg Med J 2014;31:899-903.

14. Moon JM, Chun BJ. Predicting acute complicated glyphosate intoxication in the emergency department. Clin Toxicol 2010;48:71824.

15. Watson WA, Bradford DC, Veltri JC. The volume of a swallow: correlation of deglutition with patient and container parameters. Am J Emerg Med 1983;1:278-81.

16. Lawless HT, Bender S, Oman C, Pelletier C. Gender, age, vessel size, cup vs. straw sipping, and sequence effects on sip volume. Dysphagia 2003;18:196-202. 
17. Hughes TA, Wiles CM. Clinical measurement of swallowing in health and in neurogenic dysphagia. QJM 1996;89:109-16.

18. Chee C, Arshad S, Singh S, Mistry S, Hamdy S. The influence of chemical gustatory stimuli and oral anaesthesia on healthy human pharyngeal swallowing. Chem Senses 2005;30:393-400.

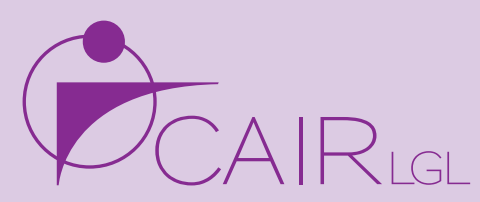

In the beginning, there was a patient...

\section{NeutraClear ${ }^{\circledR}$}

The closed system which guarantees neutral displacement and complete transparency to prevent catheter-related blood stream infections

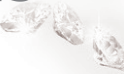

$$
\text { (1) }
$$

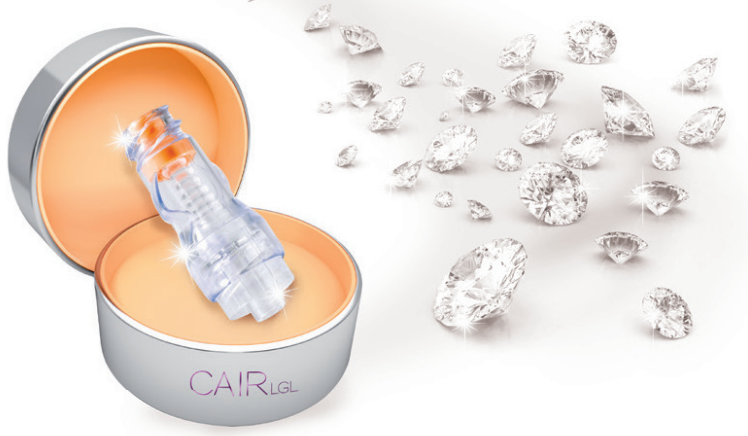

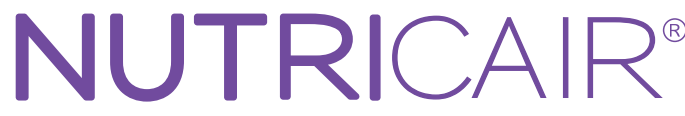

ENTERAL

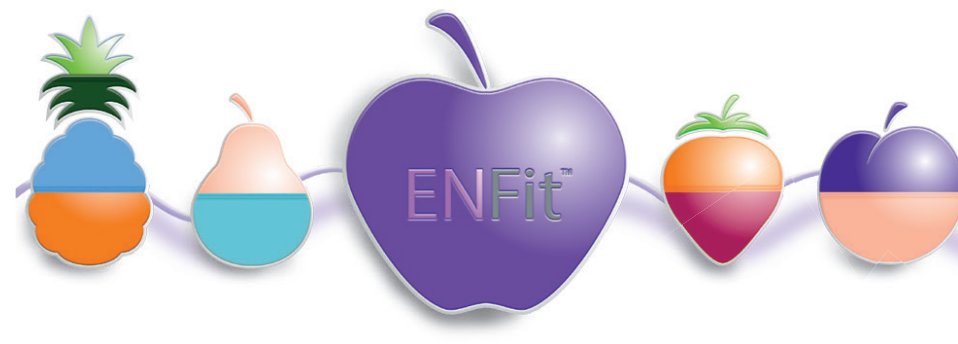

\section{Enteral nutrition} safely 